\title{
Bertrand Oligopoly Revisited
}

\author{
TÖNU PUU \\ Department of Economics, Umeå University, SE-90187, Umeå, Sweden
}

(Received 10 February 2000; In final form 9 August 2000)

\begin{abstract}
This paper reconsiders Bertrand duopoly and oligopoly in the spatial formulation due to Hotelling, 1929. The equilibrium configurations of price and location structure are considered, given elastic demand, and a full dynamics is formulated in order to check for stability of equilibrium and the possibilities of complex dynamics, such as occurs easily with Cournot oligopoly. The main discussion concerns Hotelling's original case of two sellers on a given interval, though results for different cases, such as three firms on a circle, and lattices in 2D are indicated.
\end{abstract}

Keywords: Bertrand oligopoly; Hotelling's location problem; Nonlinear maps

\section{INTRODUCTION}

An apology for the above title is in place. When Cournot in 1838 published his seminal work on duopoly, it seems not to have been commented at all for the very long period of 45 years. Then, in 1883, Bertrand published a belated and more than normally venomous review of Cournot's work (and of Wálrás's more recent work in one). Schumpeter says that, though "Bertrand was ... the first to make an attack ... it was so inadequate..." that it is in doubt whether it "would have made much impression if ... others had not ... repudiated Cournot's solution". Schumpeter says the criticism was "eagerly seized upon, as an authoritative condemnation, by people who understood neither mathematics nor economics". As other scientists, especially Edgeworth in 1897 and Hotelling in 1929, gave substance to the argument, unlike Bertrand without misinterpreting Cournot, it seems a bit displaced to continue using the denomination "Bertrand Oligopoly", but, as it happens all too often in science, the attribution is generally accepted, and we better have to continue the tradition in order to facilitate cross-referencing.

The point of controversy was the following: Cournot assumed the supply quantities of some homogeneous good to be the choice variables for the competitors, and put up the conditions for equilibrium, as well as the fundamentals for the dynamics in terms of the reaction functions, representing the optimal reaction with respect to its supply for one competitor, given the action of the other with respect to his. According to the criticism, given the good was homogeneous, one firm could, by undercutting the competitor's price, however slightly, always recover the entire market as its share. In the end this competition would lead 
to collusive monopoly, or to competitive pricing at the level of marginal costs.

Out of this argument the idea of product differentiation was born. Buyers would regard the commodity as heterogeneous, they would have preference for the product of one seller, and so only gradually desert their favourite brand when price differences became too pronounced. The now classical work on all this is Chamberlin's of 1932.

However, Hotelling assumed that the commodity itself was homogeneous, but that the competitors could establish bounded local monopolies, defended by transportation costs. This ingenious contribution dealt with competitive price duopoly, just as in the general literature on monopolistic competition, and in addition some intriguing location paradoxes, such as clustering in space. There has been a tendency in subsequent literature to single out these location paradoxes, "two ice cream sellers on a beach" in vulgar text book terms, whereas the oligopoly aspects were left for the general discussions of product differentiation with no explicit reference to space.

There are, however, still many interesting aspects to study in Hotelling's original setup, along the routes he pointed out himself, by introducing elastic demand, which changes the extreme location instability.

\section{GENERALITIES}

Suppose a firm is located along a 1D line at point $x_{i}$. To the left of it there is another firm at point $x_{i-1}$, to the right of it another at point $x_{i+1}$. The firms charge "mill" prices $p_{i-1} p_{i}, p_{i+1}$. Alternative monopolistic price policies, such as discriminatory pricing, including uniform delivery price as a special case, have been studied by Beckmann $(1968,1976)$ and could be applied also here.

Assume local demand at point $x$, given the good is transported from the supplier at point $x_{i}$ is given by a linear demand function:

$$
q_{i}= \begin{cases}\alpha-\beta\left(p_{i}+k\left|x-x_{i}\right|\right) & p_{i}+k\left|x-x_{i}\right| \leq \frac{\alpha}{\beta} \\ 0 & p_{i}+k\left|x-x_{i}\right|>\frac{\alpha}{\beta}\end{cases}
$$

here $k$ denotes the (constant) transportation cost per unit distance. Unlike supply which is concentrated to a discrete set of locations, demand is spread out continuously over the line at a density given by (1).

Total demand for the $i$ th firm then is

$$
Q_{i}=\int_{a_{i}}^{b_{i}}\left(\alpha-\beta\left(p_{i}+k\left|x-x_{i}\right|\right)\right) d x
$$

provided the upper alternative of (1) applies. The integral (2) then has the closed form solution:

$$
\begin{aligned}
Q_{i}= & \left(\alpha-\beta p_{i}\right)\left(b_{i}-a_{i}\right) \\
& -\frac{\beta k}{2}\left(\left(a_{i}-x_{i}\right)^{2}+\left(b_{i}-x_{i}\right)^{2}\right)
\end{aligned}
$$

here $a_{i}, b_{i}$ denote the boundary points for the market. These points are not fixed, but determined by the conditions that prices from different suppliers be equal in boundary points:

$$
\begin{aligned}
& p_{i}+k\left|a_{i}-x_{i}\right|=p_{i-1}+k\left|a_{i}-x_{i-1}\right| \\
& p_{i}+k\left|b_{i}-x_{i}\right|=p_{i+1}+k\left|b_{i}-x_{i+1}\right|
\end{aligned}
$$

As $x_{i-1}<a_{i}<x_{i}<b_{i}<x_{i+1}$, the conditions (4) yield:

$$
\begin{aligned}
& a_{i}=\frac{x_{i-1}+x_{i}}{2}+\frac{p_{i}-p_{i-1}}{2 k} \\
& b_{i}=\frac{x_{i}+x_{i+1}}{2}+\frac{p_{i+1}-p_{i}}{2 k}
\end{aligned}
$$

Note that this implies that always $b_{i}=a_{i+1}$. Substituting from (5) in (3) the latter becomes:

$$
\begin{aligned}
Q_{i}=\left(\alpha-\beta p_{i}\right) \frac{1}{2 k} & \left(k\left(x_{i+1}-x_{i-1}\right)\right. \\
& \left.+p_{i+1}+p_{i-1}-2 p_{i}\right)
\end{aligned}
$$




$$
\begin{aligned}
& -\frac{\beta}{8 k}\left(\left(k x_{i-1}-k x_{i}+p_{i}-p_{i-1}\right)^{2}\right. \\
& \left.+\left(k x_{i+1}-k x_{i}+p_{i+1}-p_{i}\right)^{2}\right)
\end{aligned}
$$

The $i$ th firm will maximize its profits: $\Pi_{i}=p_{i} Q_{i}-$ $C_{i}\left(Q_{i}\right)$, where we take a linear production cost function $C_{i}\left(Q_{i}\right)=c_{i} Q_{i}$. Thus:

$$
\Pi_{i}=\left(p_{i}-c_{i}\right) Q_{i}
$$

Maximising profits $\Pi_{i}$ means choosing price $p_{i}$ and location $x_{i}$. We could also keep locations fixed and devise just a price adjustment process, alone or combined with a relocation process on a slower time scale.

Suppose we first want to find the optimal location. Taking $\partial \Pi_{i} / \partial x_{i}=0$ and solving we get:

$$
x_{i}=\frac{x_{i-1}+x_{i+1}}{2}-\frac{p_{i-1}}{2 k}+\frac{p_{i+1}}{2 k}
$$

This makes sense: If the competitors' prices are equal, the firm locates midway in between, whereas a higher price of the competitor left or right drags the firm in that direction. Second order condition is $\partial^{2} \Pi_{i} / \partial x_{i}^{2}=-(1 / 2) \beta k\left(p_{i}-c_{i}\right)<0$. We must have $p_{i}>c_{i}$, as otherwise the firm makes no profit, so the second order condition is negative, and the location choice indeed maximises profit.

Given the firm has chosen an optimal location, we can substitute for $x_{i}$ from (8) back in the expression (6) for $Q_{i}$ and hence in (7) $\Pi_{i}=\left(p_{i}-c_{i}\right)$ $Q_{i}$. In order to attain a concise formula, define a new compound variable:

$$
\lambda_{i}=k\left(x_{i+1}-x_{i-1}\right)+p_{i-1}+p_{i+1}
$$

We can also write $\lambda_{i}=p_{i-1}+k\left(x_{i}-x_{i-1}\right)+p_{i+1}+$ $k\left(x_{i+1}-x_{i}\right)$, so $\lambda_{i}$ has the interpretation of the sum of the local prices of the commodity, including transportation costs, if transported to $x_{i}$ from the left neighbour $x_{i-1}$ and from the right neighbour $x_{i+1}$. The formula (9) is, however, more useful for stressing that $\lambda_{i}$ only depends on $x_{i-1}$ and $x_{i+1}$, but not on $x_{i}$.
With the new variable defined, the profits of the $i$ th firm become:

$$
\Pi_{i}=\frac{1}{16 k}\left(p_{i}-c_{i}\right)\left(\lambda_{i}-2 p_{i}\right)\left(8 \alpha-\beta\left(\lambda_{i}+6 p_{i}\right)\right)
$$

Differentiating (10), a cubic in $p_{i}$, with respect to $p_{i}$, and solving the resulting quadratic equation for the variable, we get two solutions, of which the following maximises profits:

$$
\begin{aligned}
p_{i}= & \frac{4}{9} \frac{\alpha}{\beta}+\frac{1}{3} c_{i}+\frac{1}{9} \lambda_{i}-\frac{1}{18} \\
& \sqrt{64\left(\frac{\alpha}{\beta}\right)^{2}-8 \frac{\alpha}{\beta}\left(6 c_{i}+5 \lambda_{i}\right)+\left(36 c_{i}^{2}-12 c_{i} \lambda_{i}+13 \lambda_{i}^{2}\right)}
\end{aligned}
$$

Second order conditions are easily checked, and show that (11) always yields a local maximum, whereas its conjugate, with the minus sign before the root expression reversed, always yields a minimum.

A dynamical process can now be put up where the $i$ th firm takes the price and location for the neighbours left and right as given. One may have any number $n$ of firms, and we could let $a_{1}, b_{n}$ be the given endpoints of the whole market, or we can let them be located on a circle so that $a_{1}=b_{n}$. Just three firms on a circle, where in each run the all firms adjust price and location to those of the right and left neighbours in the previous run, may be a good start, as would the case of two firms on a fixed interval, the original Hotelling case.

\section{CONSTRAINTS}

The linear demand function (1) has certain discontinuity problems which were not taken in account when evaluating the integral (2). Above the price $p_{i}=\alpha / \beta$, demand drops to zero. Of course, prices cannot be negative either. They must even be higher than the positive unit production $\operatorname{costs} p_{i}>c_{i}$. What was said above about maximum 
price must hold everywhere for price plus transportation cost. Hence, the highest total prices for the consumers, at the boundary points $a_{i}, b_{i}$, must not exceed $\alpha / \beta$ :

$$
\frac{\alpha}{\beta} \geq p_{i}+k\left|a_{i}-x_{i}\right| \quad \frac{\alpha}{\beta} \geq p_{i}+k\left|b_{i}-x_{i}\right|
$$

Both actually boil down to the same condition once we consider how $a_{i}, b_{i}$ and $x_{i}$ were determined in the above formulas (5) and (8):

$$
\frac{\alpha}{\beta} \geq \frac{1}{4}\left(p_{i-1}+2 p_{i}+p_{i+1}\right)-\frac{k}{4}\left(x_{i+1}-x_{i-1}\right)
$$

If we want to avoid having to deal with discontinuities, we must check that this condition holds all the time.

\section{DISJOINT MONOPOLIES}

Should the above condition (13) not be satisfied, then the market diameter $2 r_{i}$ is less than the interval available, because demand drops to zero at $\alpha-\beta p_{i}-\beta k r_{i}=0$, which yields

$$
r_{i}=\frac{1}{k}\left(\frac{\alpha}{\beta}-p_{i}\right)
$$

Then the quantity integral (2) becomes:

$$
\begin{aligned}
Q_{i} & =\int_{x_{i}-r_{i}}^{x_{i}+r_{i}}\left(\alpha-\beta\left(p_{i}+k\left|x-x_{i}\right|\right)\right) d x \\
& =2\left(\alpha-\beta p_{i}\right) r_{i}-\beta k r_{i}^{2}
\end{aligned}
$$

or, with substitution for the market radius from (14),

$$
Q_{i}=\frac{\beta}{k}\left(\frac{\alpha}{\beta}-p_{i}\right)^{2}
$$

Profits then are:

$$
\Pi_{i}=\frac{\beta}{k}\left(p_{i}-c_{i}\right)\left(\frac{\alpha}{\beta}-p_{i}\right)^{2}
$$

so, differentiating this cubic with respect to $p_{i}$, equating the derivative to zero, and solving, we get two solutions:

$$
p_{i}=\frac{\alpha}{\beta} \quad p_{i}=\frac{1}{3} \frac{\alpha}{\beta}+\frac{2}{3} c_{i}
$$

The first solution is a profit minimum and the second a maximum, in fact the well known solution to a spatial monopoly mill pricing problem in $1 \mathrm{D}$, given a linear demand function. (See Beckmann, 1968, 1976) This solution is relevant when demand drops to zero at a distance before the prices with accumulated transportation costs break even for the competitors. The result then is that the market areas of neighbouring firms no longer touch, but are isolated, possibly with intervals in between which are not served by any firm - the price would simply be too high for anybody to buy the commodity. Whether this occurs seems to be a question of how many firms crowd on a given distance, what the maximum price is, and what the marginal costs are.

We can easily express the monopoly market radius (14) in terms of production costs in stead of in terms of prices. Just substitute from the second expression of (18) in (14), and we get:

$$
r_{i}=\frac{2}{3} \frac{1}{k}\left(\frac{\alpha}{\beta}-c_{i}\right)
$$

The total space $L$ occupied by $n$ touching monopolies is:

$$
L=\sum_{i=1}^{n} 2 r_{i}=\frac{4}{3} \frac{1}{k} \sum_{i=1}^{n}\left(\frac{\alpha}{\beta}-c_{i}\right)
$$

It is hence clear that the total length $L$ of the space available must be less in order that oligopolistic competition should develop, i.e.

$$
L<\frac{4}{3} \frac{1}{k} \sum_{i=1}^{n}\left(\frac{\alpha}{\beta}-c_{i}\right)
$$




\section{MARKET RADIUS IN OLIGOPOLY}

Consider now the oligopoly case anew. Note that we can define a market radius even here. The choice rule for optimal location (8) implies that the firm always places itself in the midpoint of its market interval $\left[a_{i}, b_{i}\right]$. To see this, substitute from (8) into (5), and form the differences:

$$
\begin{aligned}
& x_{i}-a_{i}=\frac{x_{i+1}-x_{i-1}}{4}+\frac{p_{i-1}-2 p_{i}+p_{i+1}}{4 k} \\
& b_{i}-x_{i}=\frac{x_{i+1}-x_{i-1}}{4}+\frac{p_{i-1}-2 p_{i}+p_{i+1}}{4 k}
\end{aligned}
$$

The expressions being equal, we can define market radius even for oligopoly:

$$
r_{i}=\frac{x_{i+1}-x_{i-1}}{4}+\frac{p_{i-1}-2 p_{i}+p_{i+1}}{4 k}
$$

This result is based on the fact that there were other firms to the right and left, so what if $a_{i}$ or $b_{i}$ is fixed, i.e., that the firm is the leftmost or rightmost in a given interval? Then things become very different. It is no longer possible to define a market radius, because the firms do not locate in the centres of their market areas. They may even crowd in the same point.

\section{THE HOTELLING CASE}

This always happened in the original Hotelling case, with $\beta=0$. Suppose now that we just have two competitors, so $n=2$. Further, $a_{1}=-1$ and $b_{2}=1$ are fixed, so we consider the interval $[-1,1]$. We could have introduced an arbitrary length of the interval, but distance and transportation cost always stay in a ratio of reciprocity, so we do not lose anything in generality by fixing the interval from the outset. From (5) then:

$$
a_{2}=b_{1}=\frac{x_{1}+x_{2}}{2}+\frac{p_{2}-p_{1}}{2 k}
$$

Further from (3):

$$
\begin{aligned}
Q_{1}= & \left(\alpha-\beta p_{1}\right)\left(1+b_{1}\right) \\
& -\frac{\beta k}{2}\left(\left(1+x_{1}\right)^{2}+\left(b_{1}-x_{1}\right)^{2}\right) \\
Q_{2}= & \left(\alpha-\beta p_{2}\right)\left(1-a_{2}\right) \\
& -\frac{\beta k}{2}\left(a_{2}-x_{2}\right)^{2}+\left(\left(1-x_{2}\right)^{2}\right)
\end{aligned}
$$

The optimal locations can be found by differentiating (25)-(26) with respect to $x_{1}, x_{2}$ respectively, as location does not enter the multiplicative factor for profits in (7). Thus we obtain:

$$
\begin{aligned}
& x_{1}=\frac{x_{2}-4}{5}+\frac{p_{2}-3 p_{1}}{5 k}+\frac{2 \alpha}{5 \beta k} \\
& x_{2}=\frac{x_{1}+4}{5}+\frac{3 p_{2}-p_{1}}{5 k}-\frac{2 \alpha}{5 \beta k}
\end{aligned}
$$

Note that (27)-(28) are different from (8), as we now have one boundary point fixed for each firm. Also, for later use, note that if we solve the location choice Eqs. (27)-(28) as a simultaneous system, then we get $x_{1}+x_{2}=0$, and so from (24):

$$
a_{2}=b_{1}=\frac{p_{2}-p_{1}}{k}
$$

Hence in equilibrium the inner market boundary point only depends on the price difference divided by the transportation cost rate. In disequilibrium, the inner market boundary point, resulting from the location choice by the first firm, is obtained by substituting from (27) in (24):

$$
b_{1}=a_{2}=\frac{3 x_{2}-2}{5}+\frac{3 p_{2}-4 p_{1}}{5 k}+\frac{\alpha}{5 \beta k}
$$

Similarly, the choice by the second firm, obtained by substituting from (28) in (24) results in:

$$
b_{1}=a_{2}=\frac{3 x_{1}+2}{5}+\frac{4 p_{2}-3 p_{1}}{5 k}-\frac{\alpha}{5 \beta k}
$$


Equations (27) $-(28)$ and (30)-(31) can now be substituted in $(25)-(26)$, which can best stated by one single formula:

$$
\begin{aligned}
Q_{i}=\frac{\beta}{10 k}( & 6 p_{i}^{2}-4 p_{i}\left(2 \frac{\alpha}{\beta}+\lambda_{i}\right) \\
& \left.+\left(\frac{\alpha}{\beta}\right)^{2}+6 \frac{\alpha}{\beta} \lambda_{i}-\lambda_{i}^{2}\right)
\end{aligned}
$$

provided we define

$$
\lambda_{1}=k\left(1+x_{2}\right)+p_{2} \quad \lambda_{2}=k\left(1-x_{1}\right)+p_{1}
$$

From (32) we get profits:

$$
\begin{aligned}
\Pi_{i}=\frac{\beta}{10 k}\left(p_{i}-c_{i}\right) & \left(6 p_{i}^{2}-4 p_{i}\left(2 \frac{\alpha}{\beta}+\lambda_{i}\right)\right. \\
& \left.+\left(\frac{\alpha}{\beta}\right)^{2}+6 \frac{\alpha}{\beta} \lambda_{i}-\lambda_{i}^{2}\right)
\end{aligned}
$$

so, optimising (34) with respect to $p_{i}$ we obtain:

$$
\begin{aligned}
p_{i}= & \frac{4}{9} \frac{\alpha}{\beta}+\frac{3}{9} c_{i}+\frac{2}{9} \lambda_{i}-\frac{1}{18} \\
& \sqrt{36\left(\frac{\alpha}{\beta}-c_{i}\right)^{2}-24\left(\frac{\alpha}{\beta}-c_{i}\right)\left(\frac{\alpha}{\beta}-\lambda_{i}\right)+34\left(\frac{\alpha}{\beta}-\lambda_{i}\right)^{2}}
\end{aligned}
$$

Again the second order conditions are fulfilled for the solution (35).

We can now set up the Hotelling model as a dynamical system from (27) - (28) and (35):

$$
\begin{gathered}
x_{1}^{\prime}=\frac{x_{2}-4}{5}+\frac{p_{2}-3 p_{1}}{5 k}+\frac{2 \alpha}{5 \beta k} \\
x_{2}^{\prime}=\frac{x_{1}+4}{5}+\frac{3 p_{2}-p_{1}}{5 k}-\frac{2 \alpha}{5 \beta k} \\
p_{1}^{\prime}=\frac{4 \alpha}{9} \frac{\alpha}{\beta}+\frac{3}{9} c_{1}+\frac{2}{9} \lambda_{1}-\frac{1}{18} \\
\sqrt{36\left(\frac{\alpha}{\beta}-c_{1}\right)^{2}-24\left(\frac{\alpha}{\beta}-c_{1}\right)\left(\frac{\alpha}{\beta}-\lambda_{1}\right)+34\left(\frac{\alpha}{\beta}-\lambda_{1}\right)^{2}}
\end{gathered}
$$

$$
\begin{aligned}
p_{2}^{\prime}= & \frac{4}{9} \frac{\alpha}{\beta}+\frac{3}{9} c_{2}+\frac{2}{9} \lambda_{2}-\frac{1}{18} \\
& \sqrt{36\left(\frac{\alpha}{\beta}-c_{2}\right)^{2}-24\left(\frac{\alpha}{\beta}-c_{2}\right)\left(\frac{\alpha}{\beta}-\lambda_{2}\right)+34\left(\frac{\alpha}{\beta}-\lambda_{2}\right)^{2}}
\end{aligned}
$$

where the dash indicates advancing the map one period, from $t$ to $t+1$. Substitution from (33) for $\lambda_{1}, \lambda_{2}$ is taken as given.

\section{EQUILIBRIUM FOR TWO IDENTICAL FIRMS}

Let us first check out equilibrium. Then Eqs. (27)(28), and the two Eqs. (35) hold as a simultaneous system. Together with the two Eqs. (33) we have six equations in the six variables $x_{i}, p_{i}, \lambda_{i}, i=1,2$. Suppose for simplicity that the firms are identical, i.e., their marginal costs are equal, $c_{i}=c$ for $i=1$, 2. A good guess is that then in equilibrium the firms also charge the same mill prices, i.e., $p_{i}=p$ for $i=1,2$. From (27)-(28) we can then, substituting $p_{i}=p$, obtain

$$
x_{1}=-\frac{2}{3}+\frac{1}{3 k}\left(\frac{\alpha}{\beta}-p\right) \quad x_{2}=\frac{2}{3}-\frac{1}{3 k}\left(\frac{\alpha}{\beta}-p\right)
$$

As we see, $x_{1}+x_{2}=0$, so the firms locate symmetrically around the zero point, the midpoint of the whole interval $(-1,1)$, though not as a rule in the midpoints $\pm 1 / 2$ of their respective market areas. From (29) we already saw that in equilibrium with equal prices the markets are separated by the zero point.

Now substitute $x_{1}=x_{2}=0$ in (40), and note that both firms locate in the same point if and only if:

$$
\frac{\alpha}{\beta}-p=2 k
$$

holds. Also substitute $x_{1}=-0.5, x_{2}=0.5$ in (40) and note that the firms locate exactly in the middle 
of their intervals if and only if.

$$
\frac{\alpha}{\beta}-p=\frac{k}{2}
$$

This, however is the case of disjoint monopolies. To see this, substitute $L=2$ in (20), then use $c_{i}=c$ in (20) and $p_{i}=p$ in the second Eq. (18), and eliminate $p$. The result is (42). So this case represents disjoint monopolies. Otherwise the firms always locate closer together.

With infallible intuition Hotelling noted that in our case of elastic demand (not treated by himself) "the tendency ... to establish business excessively close ... will be less marked", but the competitors would "not go as far ... as public welfare would require", i.e., locating in the midpoints of their respective markets, due to the "tempting intermediate market". As a matter of fact, they would, but only in the extreme case of adjacent monopolies. However, monopoly pricing would not correspond to the demands of public welfare either, for a different but quite obvious reason. In Hotelling's original case it was possible that once the firms located in the same point, competition would take the form of price cutting even until the marginal cost level was reached - the classical competitive solution, but then the location choice would imply loss of public welfare due to excessive transportation costs.

In order to avoid both the extreme cases, monopoly and crowding in the centre, we would require $k / 2 \leq \alpha / \beta-p \leq 2 k$, i.e., maximum price must overshoot the equilibrium price by between half and double the transportation cost rate. From (40) the firms might even wish to overshoot crowding and locate on the wrong sides of the midpoint. Obviously such a case can never be an equilibrium solution.

Note that Hotelling's extreme crowding phenomenon may occur even if demand is elastic, something he seems not to have suspected. Also note that crowding never occurs if there are for instance three identical firms in equilibrium on a circle periphery, as an intermediate firm according to (8) locates halfway between its neighbours, and as in such a setup each firm always is surrounded by two competitors. So, crowding is a phenomenon due to fixed boundaries, even in Hotelling's original special case where demand is inelastic, i.e., $\beta=0$.

Next, note from (33) with (40) substituted that

$$
\lambda_{1}=\lambda_{2}=-\frac{\alpha}{3 \beta}+\frac{4}{3} p+\frac{5}{3} k
$$

So, the auxiliary variables become equal for the two identical competitors, and given also that marginal costs are equal, we get by substituting from (43) in (35), and using $c_{i}=c$

$$
\begin{aligned}
& p= \frac{2}{5} \frac{\alpha}{\beta}+\frac{3}{5} c+\frac{8}{5} k-\frac{3}{10} \\
& \sqrt{4\left(\frac{\alpha}{\beta}-c\right)^{2}-8\left(\frac{\alpha}{\beta}-c\right) k+34 k^{2}}
\end{aligned}
$$

We now obtained equilibrium price $p$ from (44) in terms of the three parameters, maximum price $a / b$, unit production cost $c$, and the transportation cost rate $k$. Then, substituting for equilibrium price, $p$, maximum price, $a / b$, and transportation cost rate $k$ into (40) we also obtain the equilibrium locations $x_{1}, x_{2}$.

\section{DYNAMICS OF THE HOTELLING CASE}

We need these equilibrium values for $p$ and $x_{1}, x_{2}$ also in order to investigate the stability of the equilibrium point. We obtain the 4 by 4 Jacobian matrix of the full dynamical system (36)-(39), using the auxiliary variables (33), by differentiating the system and then substituting the equilibrium values for $p$ and $x_{1}, x_{2}$. Some of the entries become very simple, others quite messy. We get:

$$
\frac{\partial\left(p_{1}^{\prime}, p_{2}^{\prime}, x_{1}^{\prime}, x_{2}^{\prime}\right)}{\partial\left(p_{1}, p_{2}, x_{1}, x_{2}\right)}=\left[\begin{array}{cccc}
0 & \frac{\partial p_{1}^{\prime}}{\partial p_{2}} & 0 & \frac{\partial p_{1}^{\prime}}{\partial x_{2}} \\
\frac{\partial p_{2}^{\prime}}{\partial p_{1}} & 0 & \frac{\partial p_{2}^{\prime}}{\partial x_{1}} & 0 \\
-\frac{3}{5 k} & \frac{1}{5 k} & 0 & \frac{1}{5} \\
-\frac{1}{5 k} & \frac{3}{5 k} & \frac{1}{5} & 0
\end{array}\right]
$$


In equilibrium, $p_{1}=p_{2}$ and $x_{1}+x_{2}=0$, and hence $\partial p_{1}^{\prime} / \partial p_{2}=\partial p_{2}^{\prime} / \partial p_{1}$ and $\partial p_{1}^{\prime} / \partial x_{2}+\partial p_{2}^{\prime} / \partial x_{1}=0$. From (36) - (39) and (33), in equilibrium, further $\partial p_{2}^{\prime} / \partial p_{1}=\partial p_{1}^{\prime} / \partial p_{2}, \quad \partial p_{1}^{\prime} / \partial x_{2}=k \partial p_{1}^{\prime} / \partial p_{2}, \quad$ and $\partial p_{2}^{\prime} / \partial x_{1}=-k \partial p_{1}^{\prime} / \partial p_{2}$. So, denoting the first reaction function (36) $p_{1}^{\prime}=\phi\left(p_{2}\right)$, and its derivative:

$$
\phi^{\prime}=\frac{\partial p_{1}^{\prime}}{\partial p_{2}}
$$

the Jacobian matrix (45) simplifies to:

$$
\left[\begin{array}{cccc}
0 & \phi^{\prime} & 0 & k \phi^{\prime} \\
\phi^{\prime} & 0 & -k \phi^{\prime} & 0 \\
-\frac{3}{5 k} & \frac{1}{5 k} & 0 & \frac{1}{5} \\
-\frac{1}{5 k} & \frac{3}{5 k} & \frac{1}{5} & 0
\end{array}\right]
$$

which just has one complex entry $\phi^{\prime}$. This entry can be calculated from (38) by differentiating with respect to $\lambda_{1}$ :

$\phi^{\prime}=\frac{2}{9}+\frac{1}{9} \frac{6 c-17 \lambda+11 \alpha / \beta}{\sqrt{36(\alpha / \beta-c)^{2}-24(\alpha / \beta-c)(\alpha / \beta-\lambda)+34(\alpha / \beta-\lambda)^{2}}}$

We dropped the indices, as in equilibrium we deal with equal prices and costs. We further have to substitute for $\lambda$ from (43), and for $p$ from (44). Then $\phi^{\prime}$ becomes completely determined by the parameters: maximum price $\alpha / \beta$, production cost $c$, and transportation cost $k$. But the resulting expression is by no means translucent.

On the other hand, the stability of equilibrium only depends on the value of $\phi^{\prime}$. The Jacobian determinant of (47) is:

$$
\left|\begin{array}{cccc}
0 & \phi^{\prime} & 0 & k \phi^{\prime} \\
\phi^{\prime} & 0 & -k \phi^{\prime} & 0 \\
-\frac{3}{5 k} & \frac{1}{5 k} & 0 & \frac{1}{5} \\
-\frac{1}{5 k} & \frac{3}{5 k} & \frac{1}{5} & 0
\end{array}\right|=-\frac{9}{25} \phi^{\prime^{2}}
$$

and the characteristic equation factorises into:

$$
\begin{aligned}
& \left(\lambda^{2}+\left(\phi^{\prime}-\frac{1}{5}\right) \lambda+\frac{3}{5} \phi^{\prime}\right) \\
& \left(\lambda^{2}-\left(\phi^{\prime}-\frac{1}{5}\right) \lambda-\frac{3}{5} \phi^{\prime}\right)=0
\end{aligned}
$$

Note the temporary shift of meaning for $\lambda$ to an eigenvalue. It so seems that the system, as far as stability of equilibrium is concerned, can be analysed as a composite of two two-dimensional maps. Further, we immediately see from (50), by substituting 1 and -1 for $\lambda$ that there is loss of stability, flip and cusp bifurcations respectively, at the critical parameter values $\phi^{\prime}=-2,-1 / 2,3 / 4$ and 3 .

Also, it is easy to see by computing the discriminants of the factors of (50) that the roots of the first factor are complex conjugates for $\phi^{\prime}$ in the interval $(7 / 5-4 \sqrt{3} / 5,7 / 5+4 \sqrt{3} / 5)$, and the roots of the second for $\phi^{\prime}$ in the interval $(-1-2 \sqrt{6} / 5,-1+2 \sqrt{6} / 5)$, so, except for a very tiny interval around zero, either one or the other of the factors produces complex roots. There are hence Hopf bifurcations with loss of stability when $\phi^{\prime}=5 / 3$ and $\phi^{\prime}=-5 / 3$, which fit nicely in these intervals. However, before that occurs there is a flip or cusp bifurcation due to some real eigenvalue. In conclusion the equilibrium point is completely stable in the interval $-1 / 2<\phi^{\prime}<3 / 4$.

Thus all depends on the values $\phi^{\prime}$ can take. However, inspecting (48), we find that the values it can take are bounded by the expression:

$$
\frac{2}{9}-\frac{\sqrt{34}}{18} \operatorname{sgn}(\lambda-1)
$$

so $\phi^{\prime}$ is in the interval $[-0.1017 \ldots, 0.5462 \ldots]$. We therefore conclude that the equilibrium of two identical firms on a given interval cannot lose stability, and so not produce any interesting dynamic phenomena at all.

The question now is whether this stability is due to the fact that we studied the equilibrium of identical firms. The reaction functions of the type 
(35) are of the upside down parabola shape which are in principle capable of producing chaos. However, from experience, we would need a rising branch of one reaction curve to intersect a falling branch of the other, with sufficiently high slopes to produce instability around the intersection point. For this we would need some asymmetry, and so identical firms will simply not do. The case with different production costs for the firms (the only possible point of difference), however, becomes much more complex. For instance, we cannot solve for the equilibrium prices from the system (35), because they provide a pair of second order equations, and so closed form solutions such as (44) cannot be obtained. Further, the fourth order characteristic Eq. (50) does not factorise. Still, the derivatives of the two reaction functions, such as $\phi^{\prime}$, their product to be quite exact, still controls everything. Preliminary studies indicate that the same tendency to stability remains even if the firms are unequal.

So the real problem seems to be too much linearity. The demand function is linear, and so is the cost function. In spaceless economics we would not obtain any nonlinearity at all. However, space itself produces some nonlinearity. We got cubic expressions for the profits, and quadratic equations to determine the price reactions, just from integrating over space. But obviously it was not enough, so if we want to produce complex dynamics we need to introduce some nonlinearity in the basic assumptions already. We called the proven stability a problem, but it is a matter of taste if we regard the lack of interesting dynamics a problem or not. The average economist would be more than satisfied with stability of equilibrium.

\section{THE DEGREES OF FREEDOM}

Throughout the discussion we kept the coefficients $\alpha$ and $\beta$ from the demand function, though nowhere in the reaction functions did these parameters enter except in terms of their ratio $\alpha / \beta$, so we could just have used one symbol for their ratio. Further, inspecting the reaction formulas it is obvious that if we redefine all value variables (prices, production costs, transportation costs) as ratios to this maximum price $\alpha / \beta$, then nothing at all is changed. Accordingly, we could even put $\alpha / \beta=1$ without any loss of generality. In this way the free parameters are reduced to only two, production costs $c$, and transportation costs $k$.

\section{DIFFERENT MODELS}

There are different variants of the original Hotelling model, which may be worthwhile studying, both for their own interest, and for the possibility that they may produce complex dynamics. One has been touched upon already: the case of three oligopolists on a closed curve. The distinguishing factor is that there are no boundary points, so each firm is surrounded by two competitors, and a phenomenon such as crowding never occurs.

Another obvious generalisation is to 2D, touched upon by Hotelling already, which is the really interesting case. However it is incomparably more complex, at least for a study of the full dynamics. In 2D, market boundaries become curves, so market areas have shape in addition to size. Also, in $2 \mathrm{D}$, there arises a question of the distance metric to be applied for the transportation cost, the Euclidean metric by no means being the analytically easiest, nor the factually most relevant. Candidates for equilibrium obviously are the regular lattices, corresponding to square, triangular, and hexagonal Eulerian tessellations of market areas. General considerations of transversality already would seem to favour the hexagonal case, singled out by classical location theory, though on grounds of the economy in its compactness. It is highly interesting to work out the stability problems for the various equilibrium tessellations. The simplest case to treat is the regular square tessellation, especially if combined with a so called "Manhattan metric" where 
equidistance loci too are not circular but square. Before leaving these conjectures, we note that in $2 \mathrm{D}$ the degree of nonlinearity is automatically raised, profit functions becoming quartic, and reaction functions roots to cubic equations.

\section{References}

Beckmann, M. J. (1968) Location Theory (Random House, New York).

Beckmann, M. J. (1976) "Spatial Price Policies Revisited", Bell Journal of Economics, 7, 619-630.

Beckmann, M. J. and Thisse, J. F. (1986) "The Location of Production Activities", Handbook of Regional and Urban Economics, 1, 21-95 (Elsevier Science).

Bertrand, J. (1883) "Théorie Mathématique de la Richesse Sociale", Journal des Savants, 48, 499-. 508.

Chamberlin, E. H. (1932) The Theory of Monopolistic Competition (Harvard University Press).
Cournot, A. (1838) Récherches sur les Principes Mathémaiques de la Théorie des Richesses (Hachette, Paris).

Edgeworth, F. Y. (1897) "La Teoria Pura del Monopolio", Giornale degli Economisti, 15, 13.

Greenhut, M. L. and Ohta, H. (1972) "Monopoly Output under Alternative Spatial Pricing Techniques", American Economic Review, 62, 705-713.

Hotelling, H. (1929) "Stability in Competition", Economic Journal, 41, 41-57.

Palander, T. F. (1935) Beiträge zur Standortstheorie (Almqvist \& Wiksell, Uppsals).

Ponsard, C. (1954) Économie et Espace - essai d'integration du facteur spatial dans l'analyse économique (Sedes, Paris).

von Stackelberg, H. (1938) Probleme der unvollkommenen Konkurrenz, Weltwirtschaftliches Archiv, 48, 95.

Schumpeter, J. A. (1954) History of Economic Analysis (Oxford University Press).

Smithies, A. (1941) "Monopolistic Price Policy in a Spatial Market", Econometrica, 9, 63-73.

Smithies, A. (1941) "Optimum Location in Spatial Competition", Journal of Political Economy, 49, 423-439.

Sraffa, P. (1926) "The Laws of Returns Under Competitive Conditions", The Economic Journal, 36, 535-550. 


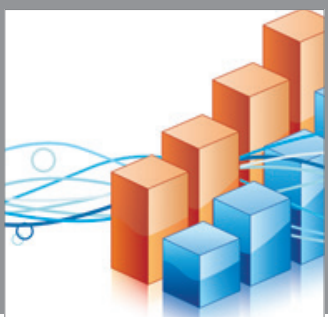

Advances in

Operations Research

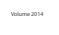

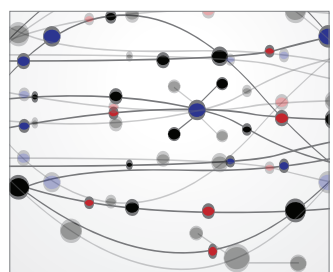

\section{The Scientific} World Journal
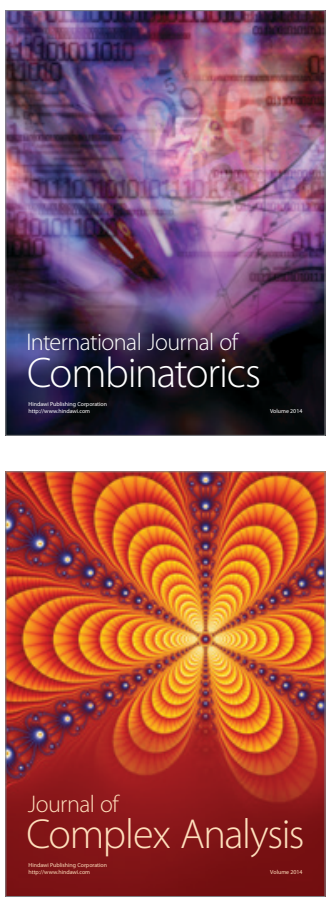

International Journal of

Mathematics and

Mathematical

Sciences
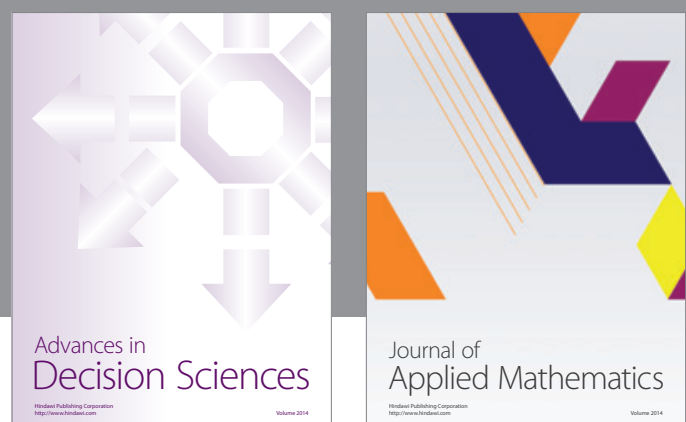

Journal of

Applied Mathematics
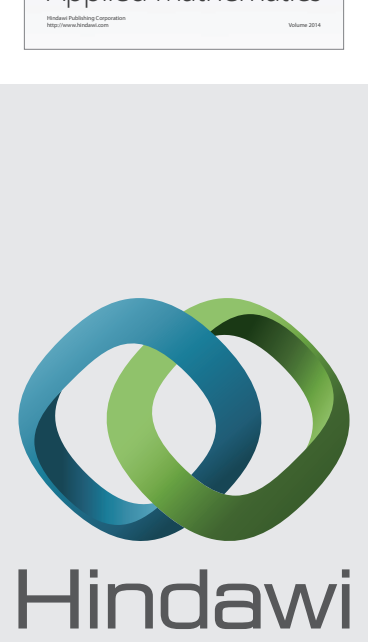

Submit your manuscripts at http://www.hindawi.com
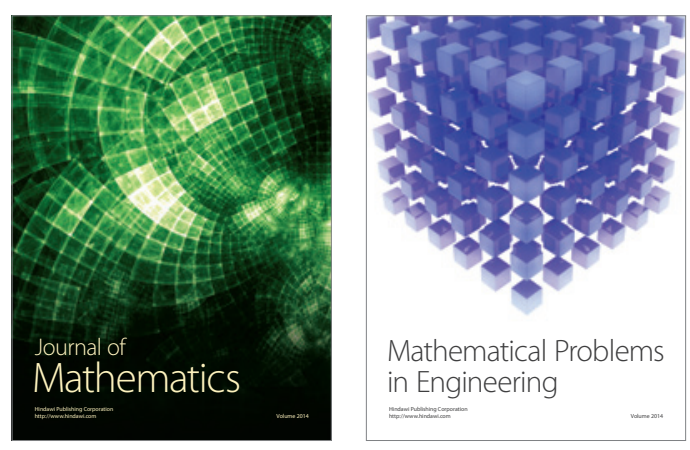

Mathematical Problems in Engineering
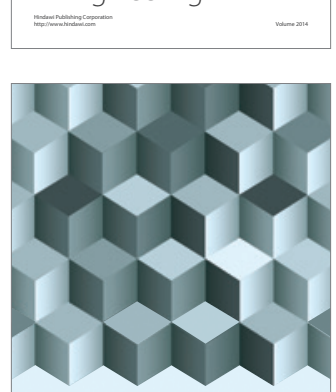

Journal of

Function Spaces
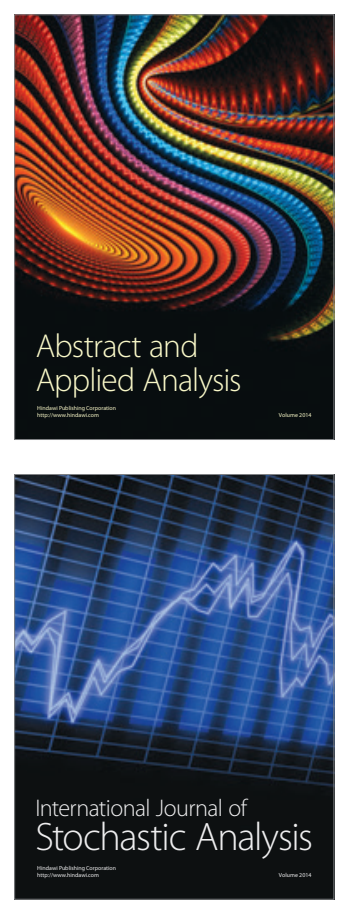

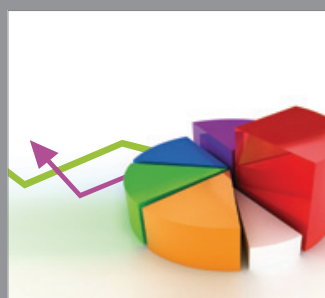

ournal of

Probability and Statistics

Promensencen
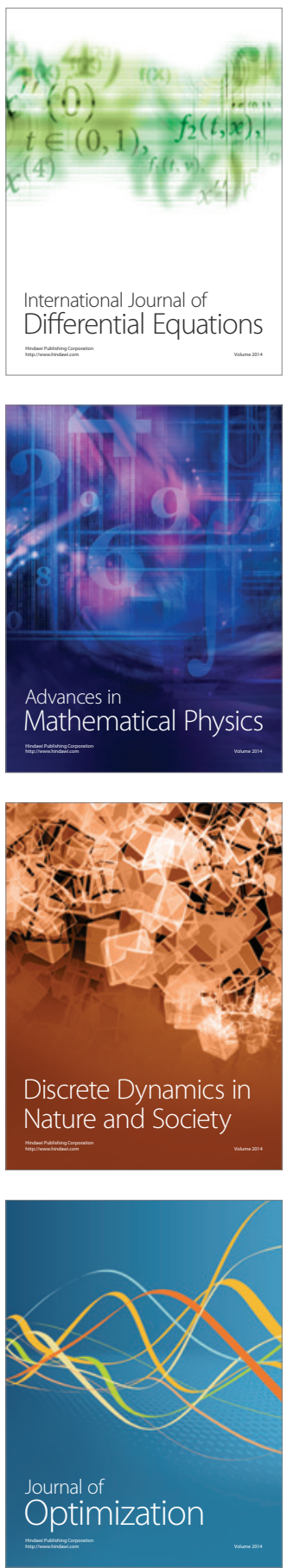